\title{
Procesos socioemocionales de estudiantes universitarios por medidas sanitarias COVID-19: resultados preliminares ${ }^{1}$
}

\section{Socio-emotional processes of university students regarding sanitary measures for COVID-19: preliminary results ${ }^{2}$}

DOI: $10.46932 / \mathrm{sfjdv2n3-028}$

Received in: May 1st, 2021

Accepted in: Jun 30th, 2021

\section{Oliva López Sánchez}

Highest Academic Background/Máxima formación académica: Doctorado en Antropología Institution/Institución: Facultad de Estudios Superiores Iztacala de la Universidad Nacional Autónoma de México

Address/Dirección: Av. De los Barrios No. 1 Los Reyes Iztacala, Tlalnepantla, Estado de México, CP 54090

E-mail/Correo electrónico: olivalopez@unam.mx

\section{Xamanek Cortijo Palacios}

Highest Academic Background/Máxima formación académica: Doctorado en Neuroetología Institution/Institución: Instituto Interdisciplinario de Investigaciones de la Universidad de Xalapa. Xalapa, Veracruz, México

Addres/Dirección: Km 2. Carretera Xalapa-Veracruz, No.341 Col. Acueducto Ánimas. C.P. 91190 E-mail/Correo electrónico: 1xcortijo@gmail.com

\section{RESUMEN}

Objetivos. Identificar y analizar los procesos socioemocionales de estudiantes de nivel superior derivados de las medidas sanitarias por COVID-19. Método. Investigación exploratoria, descriptiva, transversal de corte cuantitativo, realizada en una comunidad estudiantil $(n=3194)$. Mediante un cuestionario en línea se indagaron los efectos psicosociales y procesos socioemocionales generados por el distanciamiento social. El incremento y disminución de las emociones antes y después del inicio de la jornada de sana distancia se analizó con la prueba t de Student. Resultados. Antes del COVID-19 se reportaron emociones de bienestar y malestar. Antes y durante el distanciamiento por COVID-19 se reportaron emociones de bienestar y malestar, observándose diferencias significativas $(p<0.05)$ en todas las emociones evaluadas con excepción de estrés (mujeres $t=0.331 ; p=0.740$; hombres $t=1.297 ; p=0.197$ ), ansiedad (diversa $t$ $=2.666 ; p=0.183$ ) y depresión (diversa $t=1.30958 ; p=0.195$ ). La situación financiera personal y familiar genera mayor preocupación en la población (70\%) y destaca como estrategia para mejorar el ánimo el consumo de bebidas alcohólicas y tabaco en hombres (30 a 32 años; 46\%). Discusión y

\footnotetext{
${ }^{1}$ El artículo es un producto directo del Proyecto: "Características psicosociales y procesos socioemocionales en comunidades estudiantiles universitarias frente al aislamiento sanitario por COVID 19: hacia una política institucional del cuidado, autocuidado y autoatención" financiado por el Programa de Apoyo a Proyectos de Investigación e Innovación Tecnológica (PAPIIT) de la Dirección General del Personal Académico de la Universidad Nacional Autónoma de México (DGAPAUNAM), registro IN301021. El proyecto también cuenta con el número de registro 1362 del Comité de Ética de la Facultad de Estudios Superiores Iztacala de la UNAM.

2 The article is a product of the Project: "Características psicosociales y procesos socioemocionales en comunidades estudiantiles universitarias frente al aislamiento sanitario por COVID 19: hacia una política institucional del cuidado, autocuidado y autoatención" The research is funded by Program for Research and Technological Innovation Projects ( PAPIIT) of the Dirección General del Personal Académico de la Universidad Nacional Autónoma de México (DGAPA-UNAM) IN301021. The project also has the registration number 1362 of the Ethics Committee of the Facultad de Estudios Superiores Iztacala UNAM.
} 
conclusión. El análisis del incremento de malestar emocional en la población estudiantil en el distanciamiento social por COVID-19 requiere incluir las determinantes sociales y los componentes socioculturales de las emociones para explicar los procesos socioemocionales ante situaciones de emergencia.

Palabras clave: COVID-19, emociones, estudiantes, aislamiento social, factores sociológicos, salud mental (fuente: DeCS, BIREME).

\begin{abstract}
Objectives. Identify and analyze the socio-emotional processes of university students resulting from social distancing measures for COVID-19. Method. Exploratory, descriptive, quantitative cross-sectional research carried out in a student community $(n=3,194)$. Psychosocial effects and socio-emotional processes generated by social distancing were investigated through the use an online questionnaire. The increase and decrease in emotions before and after the start of the Healthy Distance Days was analyzed with Student's t-test. Results. Before and during COVID-19 distancing emotions of wellbeing and discomfort were reported, a significant difference $(\mathrm{p}<0.05)$ was observed in all the emotions evaluated with the exceptions of stress (women $\mathrm{t}=0.331 ; \mathrm{p}=0.740 ;$ men $\mathrm{t}=1.297 ; \mathrm{p}=0.197$ ), anxiety (diverse $\mathrm{t}=$ $2.666 ; p=0.183$ ) and depression (diverse $t=1.30958 ; p=0.195$ ). Personal and family financial situations generate greater concern in the population $(70 \%)$ and the consumption of alcoholic beverages and tobacco in men (30 to 32 years; $46 \%$ ) stands out as a strategy to improve mood. Discussion and Conclusion. The analysis of the increase in emotional distress in the student population during social distancing for COVID-19 requires including the social determinants and the socio-cultural components of emotions to explain the socio-emotional processes in emergency situations.
\end{abstract}

Key words: COVID-19, emotions, students, social isolation, sociological factors, pandemic, mental health (source: MeSH, NLM).

\title{
1 INTRODUCCIÓN
}

La aparición del virus SARS-CoV2 (COVID-9) en el mes de diciembre de 2019 en Wuhan China y la acelerada expansión en Europa, América Latina, Norteamérica y África durante los primeros meses del 2020 conllevó a la implementación de distintas medidas sanitarias a nivel global: 1) distanciamiento social para el control de la asistencia a lugares concurridos para restringir la interacción entre personas procurando una distancia física; 2) aislamiento social que refiere la separación física de personas contagiadas de las sanas; 3) cuarentena obligatoria y voluntaria (Sánchez-Villena, 2020). La cuarentena es la práctica sanitaria de aislamiento más antigua que data de la Italia del siglo XIV; alude a la antigua práctica de los cordones sanitarios impuestos en los puertos marítimos para controlar la incubación de enfermedades contagiosas (Ledermann, 2003). Desde entonces el término cuarentena se ha empleado para denominar al periodo de contagio de las enfermedades.

La cuarentena se utiliza como estrategia sanitaria para separar y restringir el movimiento de personas que potencialmente pueden estar infectadas y que no presentan síntomas (Mendoza-Velásquez, 2020). Estas pueden variar en el número de días de aislamiento o restricción de movilidad forzada y 
voluntaria. En el caso de México, se implementó una estrategia de distanciamiento social anunciado por el Gobierno Federal y la secretaría de Salud para contener el riesgo de contagio del COVID-19 bajo la Jornada de Sana Distancia. La restricción de movilidad fue voluntaria, inició el 23 de marzo del año 2020 y se ha mantenido en función de la semaforización epidemiológica que regula las actividades esenciales y no esenciales en la modalidad presencial.

Las cuarentenas, los confinamientos y distanciamientos sociales como medidas de control sanitario suelen ser experiencias desagradables para quienes las padecen, por la separación de sus actividades y sus vínculos socioafectivos, por la pérdida de libertad, la incertidumbre y el aburrimiento (MendozaVelásquez, 2020). La Organización Mundial de la Salud y su filial en las Américas la Organización Panamericana de la Salud (OPS) advirtieron sobre la importancia de atender la salud mental a causa de la prolongada cuarentena. En Europa y América Latina existen reportes de incrementos de crisis de ansiedad, pánico, depresión e incluso sobre intentos y suicidios consumados (Mendoza-Velásquez, 2020; PiñaFerrer, 2020; Riesgo, 2020; Brooks et al., 2020; Rubin \& Wessely, 2020).

Las investigaciones sobre los efectos psicoemocionales por las medidas sanitarias implementadas para contener la pandemia COVID-19 son escasas y se han llevado a cabo a partir de metodologías cuantitativas. Se tienen algunas estimaciones y protocolos generales de las crisis psicológicas de emergencia en los pacientes y familiares por la neumonía causada por el SARS-CoV2, casos sospechosos, personal de salud que atiende poblaciones infectadas y público en general (Mendoza-Velásquez, 2020). Investigaciones en el departamento de psicología del King College London en el Reino Unido en el mes de enero del 2020, a dos meses de los inicios de la pandemia en Wuhan, advirtieron de los efectos psicológicos adversos como pánico y ansiedad por la falta de claridad e información acerca de la pandemia COVID-19. La muerte de los primeros casos de enfermos, la posible escasez de alimentos, los rumores sobre la pandemia y la cuarentena fueron los escenarios que podían detonar alteraciones psicológicas (Rubin, 2020).

De acuerdo con el estudio de Rubin, en regiones como Taiwán en donde la cuarentena fue obligatoria, reporta los efectos psicológicos generados por el aislamiento del personal de salud del hospital municipal de Hoping en Taipei tras un encierro por dos semanas que "causó una sensación de histeria colectiva, impulsando al personal a medidas desesperadas" (2020, p. 1). Estudios posteriores señalan los costos psicológicos por el prolongado aislamiento social y por la cuarentena (Brooks et al., 2020). Entre los efectos psicológicos negativos, de acuerdo con bases de datos electrónicas, se identificaron el estrés postraumático, confusión, enojo, miedo y la ansiedad. A mayor tiempo de cuarentena mayores los trastornos mentales y emocionales (Mendoza-Velásquez, 2020). Los factores estresores se asocian con la duración de la cuarentena, el miedo a la infección, la frustración y el aburrimiento, ausencia de suministros 
y servicios de salud información médica no verificada, problemas económicos y estigmas sociales por contraer la enfermedad (Brooks et al., 2020).

Estudios de diseño bibliográfico señalan que la cuarentena obligatoria y prolongada ha conllevado a episodios de "desesperación, perdida de libertad, aburrimiento, insomnio, poca concentración e indecisión, irritabilidad, ira, ansiedad, angustia, de no tener contacto físico con familiares, amigos, no poder desarrollar una rutina de vida normal” (Piña-Ferrer, 2020, p. 195).

Análisis más detallados de las alteraciones psicológicas mentales y emocionales, por causa de la prolongada cuarentena, el distanciamiento y aislamiento social, requieren tomar en cuenta las variables psicosociales como sexo, adscripción étnico-racial, nivel de ingresos, nivel socioeducativo, accesos a bienes y servicios (Medina-Mora et al., 2015; Rodríguez-Yunta, 2016; Urbina, 2016; Sol-Pastorino et al., 2017). Para una comprensión más amplia del impacto de las diferencias estructurales en la vida emocional y mental de las personas en momentos de emergencia como la pandemia por COVID-19 es necesario implementar investigaciones exploratorias como la desarrollada en este trabajo. Tal es el caso de perspectivas que incluyan aspectos psicológicos, sociales y culturales para la explicación de los procesos socioemocionales (Thoits, 1990; Bericat, 2018; López, 2019). Los cuales refieren el manejo que las personas hacen de sus emociones en función de su posición social estrechamente relacionada con su identidad sexo/género, generacionales, de estratificación social, educativas y sus condiciones vitales (López, 2020). Por lo tanto, estos procesos permiten identificar la relación entre las emociones resultantes de las medidas de contención sanitaria: distanciamiento social, asilamiento social y cuarentena por COVID-19 con las variables identitarias y socioeducativas de los actores sociales. Por lo tanto, el objetivo del estudio es analizar los procesos socioemocionales de estudiantes universitarios derivados de las medidas de control sanitario en la Jornada de Sana Distancia implementada por la Secretaría de Salud en México por COVID-19.

\section{MÉTODO}

\subsection{DISEÑO DEL ESTUDIO}

Esta investigación es exploratoria, descriptiva, transversal de corte cuantitativo.

\subsubsection{Descripción de la muestra y lugar del estudio}

El estudio de la población se realizó del 28 de abril al 1 de junio de 2020, el cual de manera inicial incluía una muestra no probabilística de 3427 estudiantes de nivel superior de la Facultad de Estudios Superiores campus Iztacala de la Universidad Nacional Autónoma de México (FES-Iztacala UNAM). Sin embargo, fueron eliminadas las respuestas de 227 participantes por no contener la información completa 
en el instrumento de evaluación, siendo este el único criterio de exclusión, con lo cual se obtuvo una muestra final de 3194 cuestionarios respondidos en su totalidad (criterio de inclusión) por estudiantes y pasantes de las carreras de Psicología, Medicina, Enfermería, Biología, Optometría, Odontología y de nivel posgrado (maestría y doctorado). Los cuales fueron divididos para su posterior análisis por sexo (mujeres y hombres) e identidad de género (diversa), así como también por rangos de edad: 18-20, 2123, 24-26, 27-29, 30-32, 33 y más años, con la finalidad de explorar la influencia de esas características en las respuestas de los participantes. Es importante mencionar, que si bien la información sobre "identidad diversa" que se analiza es limitada por el número de participantes, se tomó la decisión de conservarla para respetar el criterio ético de inclusión y no discriminación a la diversidad sexual.

\section{MEDICIONES}

Mediante un cuestionario en formato de encuesta en línea, se indagaron los efectos psicosociales y los procesos socioemocionales generados por las medidas de control sanitario por COVID-19 de las primeras siete semanas de distanciamiento social en el marco de la jornada sanitaria. El cuestionario denominado Medidor Psicosocial y Socioemocional frente a la contingencia COVID-19 (MPE-COVID19), compuesto por 30 ítems divididos en seis secciones, las cuales exploran: 1) Datos demográficos en esta sección se registraron el sexo y la identidad de género, la edad (rangos), grado escolar, nivel educativo y carrera a la pertenecían cada uno de los participantes, 2) Espacios y convivencia familiar, 3) Salud, alimentación e higiene del sueño, 4) Salud y vida emocional, 5) Actividades académicas, 6) Dinámicas en casa antes/durante COVID-19. El instrumento cierra con dos preguntas abiertas que permitió al estudiantado dejar sus comentarios y requerimientos de apoyo psicológico. Este trabajo es un primer acercamiento analítico que pretende un abordaje interdisciplinario de un fenómeno social de alta relevancia en el contexto de la pandemia COVID 19.

Con el propósito de determinar la forma en que se ha experimentado la pandemia desde el ámbito social y emocional, fue seleccionada la sección cuatro: Salud y vida emocional para su análisis en el presente estudio. Incluye cinco cuestionamientos: 1. Antes del confinamiento, tus estados emocionales habituales eran... 2. ¿Qué emociones has experimentado durante las semanas del confinamiento social por el COVID-19? 3. ¿Cuáles son los escenarios que más preocupación e incertidumbre te generan las medidas de contingencia por COVID-19? Y 4. ¿Qué haces para mejorar tus estados de ánimo? (López, 2020). 


\section{PROCEDIMIENTO}

Utilizando las redes sociales de la FES-Iztacala UNAM se invitó a toda la comunidad estudiantil a participar en la evaluación del MPE-COVID-19. La cual se llevó a cabo usando la plataforma Google Formularios, donde de manera autoaplicada la comunidad de jóvenes respondieron el instrumento. Esta metodología empleada favorece la reducción de sesgos en la información y selección de los participantes, debido a la poca injerencia del investigador en el proceso de reclutamiento, evaluación y registro de datos. Además, se consideró para su posterior análisis solamente a aquellos que cumplieron con el criterio de inclusión.

\section{ANÁLISIS ESTADÍSTICO}

Fueron analizados los resultados sociodemográficos y las cinco preguntas de la sección cuatro correspondiente a Salud y vida emocional del MPE-COVID-19. Estos se presentan con las frecuencias y porcentajes de cada una de las variables evaluadas: estados emocionales previos y durante las medidas sanitarias de distanciamiento social, los escenarios sociales y personales de mayor preocupación e incertidumbre generados por las medidas por COVID-19 y actividades para mejorar el ánimo. Las variables antes mencionadas fueron diferenciadas por sexo/identidad de género, rango de edad y carrera según corresponda.

Con la finalidad de comparar el incremento y disminución de las emociones antes y después del inicio del distanciamiento social, se utilizó una prueba $t$ de Student para muestras relacionadas y comparaciones múltiples de Holm-Sidak donde se consideró un nivel de significancia estadística de $p<$ 0,05, para lo cual se empleó el programa estadístico IBM SPSS Statistics 21. Fueron graficadas las medias \pm EEM. Por otro lado, el marco teórico de los estudios socioculturales de las emociones permitió incorporar las variables sociales para analizar cualitativamente su relación con los procesos emocionales ante las medidas sanitarias de control por la pandemia de COVID-19 (López, 2011).

\section{CONSIDERACIONES ÉTICAS}

El proyecto del cual se originaron los datos analizados cuenta con la aprobación del Comité de Ética de la Facultad de Estudios Superiores Iztacala de la UNAM (número de registro 1362). La presente investigación se clasifica dentro de la Ley General de Salud en Materia de Investigación para la Salud (Ley General de Salud, 2020, Artículo 79) y la Norma Oficial Mexicana 012-SSA3-2012 (2013) en la categoría II, por tratarse de procedimientos de uso común y seguros. Este estudio fue conducido conforme a los principios de la declaración de Helsinki (Asociación Médica Mundial, 2013) y la Ley Federal de 
Protección de Datos Personales en Posesión de los Particulares (2010). Se conservó rigurosamente el anonimato de todos y cada uno de los participantes.

\section{RESULTADOS}

\subsection{CARACTERÍSTICAS SOCIODEMOGRÁFICAS Y ESCOLARES DE LOS PARTICIPANTES}

Se obtuvo una muestra de 3194 estudiantes de licenciatura, pasantes y posgrado, donde el 69\% fueron mujeres y el 30\% hombres, el resto de la población se definió con identidad diversa (1\%). Las carreras que más participaron en el estudio fueron odontología (29\%), psicología (25\%), biología (22\%) y en menor proporción estudiantes en condición de pasantes $(6 \%)$ y de nivel posgrado (3\%). Los jóvenes de entre 18 a 20 y 21 a 23 años representan el 82\% de la población analizada (Tabla 1).

Tabla 1. Características sociodemográficas y escolares de los participantes

\begin{tabular}{|c|c|c|c|c|c|c|c|c|c|c|c|c|c|c|c|c|c|}
\hline \multirow[t]{2}{*}{$\begin{array}{c}\text { Sexo/ } \\
\text { Identidad }\end{array}$} & \multirow{2}{*}{$\begin{array}{l}\text { Edad } \\
\text { rango } \\
\end{array}$} & \multicolumn{2}{|c|}{$\begin{array}{c}\text { Odontología } \\
(\mathbf{n}=\mathbf{9 2 7})\end{array}$} & \multicolumn{2}{|c|}{$\begin{array}{c}\text { Psicología } \\
(\mathrm{n}=\mathbf{8 1 3})\end{array}$} & \multicolumn{2}{|c|}{$\begin{array}{c}\text { Biología } \\
(\mathbf{n}=707)\end{array}$} & \multicolumn{2}{|c|}{$\begin{array}{c}\text { Enfermería } \\
(\mathrm{n}=\mathbf{3 5 9})\end{array}$} & \multicolumn{2}{|c|}{$\begin{array}{c}\text { Medicina } \\
(\mathrm{n}=79)\end{array}$} & \multicolumn{2}{|c|}{$\begin{array}{c}\text { Optometría } \\
(\mathrm{n}=14)\end{array}$} & \multicolumn{2}{|c|}{$\begin{array}{l}\text { Pasantes } \\
(n=196)\end{array}$} & \multicolumn{2}{|c|}{$\begin{array}{l}\text { Posgrado } \\
(\mathbf{n}=99)\end{array}$} \\
\hline & & $n$ & $\%$ & $n$ & $\%$ & $n$ & $\%$ & $n$ & $\%$ & $n$ & $\%$ & $n$ & $\%$ & $n$ & $\%$ & $n$ & $\%$ \\
\hline \multirow[t]{6}{*}{ Mujeres } & $18-20$ & 443 & 47,7 & $\begin{array}{c}23 \\
0\end{array}$ & 28,2 & $\begin{array}{c}20 \\
9\end{array}$ & 29,5 & 143 & 39,8 & 22 & 27,8 & 1 & 7,1 & 1 & 0,5 & - & - \\
\hline & $21-23$ & 181 & 19,5 & $\begin{array}{c}21 \\
7\end{array}$ & 26,6 & $\begin{array}{c}17 \\
4\end{array}$ & 24,6 & 109 & 30,3 & 35 & 44,3 & 5 & 35,7 & 57 & 29,0 & 1 & 1,0 \\
\hline & $24-26$ & 22 & 2,3 & 52 & 6,4 & 37 & 5,2 & 11 & 3,0 & 3 & 3,8 & 1 & 7,1 & 50 & 25,5 & 7 & 7,0 \\
\hline & $27-29$ & 5 & 0,5 & 18 & 2,2 & 7 & 0,9 & 7 & 1,9 & 1 & 1,2 & - & - & 13 & 6,6 & 10 & 10,1 \\
\hline & $30-32$ & 1 & 0,1 & 12 & 1,4 & 3 & 0,4 & - & - & 2 & 2,5 & - & - & 8 & 4,0 & 10 & 10,1 \\
\hline & $\begin{array}{l}33 \text { y } \\
\text { más }\end{array}$ & 1 & 0,1 & 31 & 3,8 & 2 & 0,2 & 3 & 0,8 & - & - & 1 & 7,1 & 11 & 5,6 & 48 & 48,4 \\
\hline \multirow[t]{6}{*}{ Hombres } & $18-20$ & 165 & 17,8 & 93 & 11,4 & $\begin{array}{c}11 \\
2\end{array}$ & 15,8 & 38 & 10,5 & 6 & 7,5 & - & - & - & - & - & - \\
\hline & $21-23$ & 81 & 8,7 & $\begin{array}{c}10 \\
7\end{array}$ & 13,1 & $\begin{array}{c}11 \\
8\end{array}$ & 16,6 & 37 & 10,3 & 7 & 8,8 & 4 & 28,5 & 17 & 8,6 & 1 & 1,0 \\
\hline & $24-26$ & 17 & 1,8 & 18 & 2,2 & 23 & 3,2 & 4 & 1,1 & 2 & 2,5 & 2 & 14,2 & 19 & 9,6 & 2 & 2,0 \\
\hline & $27-29$ & 2 & 0,2 & 13 & 1,6 & 8 & 1,1 & 2 & 0,5 & - & - & - & - & 8 & 4,0 & 4 & 4,0 \\
\hline & $30-32$ & 3 & 0,3 & 4 & 0,4 & 1 & 0,1 & - & - & - & - & - & - & 5 & 2,5 & 2 & 2,0 \\
\hline & $\begin{array}{l}33 \text { y } \\
\text { más }\end{array}$ & 1 & 0,1 & 11 & 1,3 & 2 & 0,2 & 1 & 0,2 & - & - & - & - & 7 & 3,5 & 14 & 14,1 \\
\hline \multirow[t]{4}{*}{ Diversa } & $18-20$ & 4 & 0,4 & 5 & 0,6 & 8 & 1,1 & 4 & 1,1 & 1 & 1,2 & - & - & - & - & - & - \\
\hline & $21-23$ & - & - & 1 & 0,1 & 3 & 0,4 & - & - & - & - & - & - & - & - & - & - \\
\hline & $24-26$ & - & - & 1 & 0,1 & - & - & - & - & - & - & - & - & - & - & - & - \\
\hline & 27- 29 & 1 & 0,1 & - & - & - & - & - & - & - & - & - & - & - & - & - & - \\
\hline
\end{tabular}

$\mathrm{n}=$ número de participantes; \% = porcentaje

7.2 ESTADOS EMOCIONALES HABITUALES ANTES Y DURANTE EL DISTANCIAMIENTO POR COVID-19

La comparación entre la presencia, incremento o disminución de las emociones durante las primeras semanas de distanciamiento social permitió establecer diferencias en los tres grupos explorados (mujeres, hombres e identidad diversa). En el caso de las mujeres, se observó de manera inicial una mayor presencia de tranquilidad, felicidad, confianza, gratitud, atención y estrés; y en menor proporción tristeza, ansiedad y preocupación. Posterior a las primeras semanas de distanciamiento social se determinaron 
diferencias significativas $(p<0,05)$ en todas las emociones evaluadas con excepción de estrés $(t=0,331$, $p=0,740$; Figura 1a).

Los hombres antes de COVID-19 reportaron sentir tranquilidad, felicidad, confianza y atención como algunas de las emociones más frecuentes, seguido de ansiedad, irritación y estrés. Al comparar el incremento o disminución de las emociones examinadas, transcurridas las primeras semanas de la jornada sanitaria, se establecieron diferencias significativas $(p<0,05)$ en todas con excepción del estrés el cual mantuvo una constante ( $t=1,297 ; p=0,197$; Figura $1 \mathrm{~b})$.

Por último, los estudiantes con identidad diversa expresaron en su mayoría antes del distanciamiento social tranquilidad, felicidad, preocupación, depresión y estrés. Al contrastar las emociones con las reportadas durante el COVID-19, todas presentaron diferencias significativas excluyendo a la ansiedad $(t=2,666 ; p=0,183)$ y la depresión $(t=1,309 ; p=0,195$; Figura 1c).

Figura 1. Estados emocionales habituales antes y durante el distanciamiento por COVID-19.

Mujeres

(a)

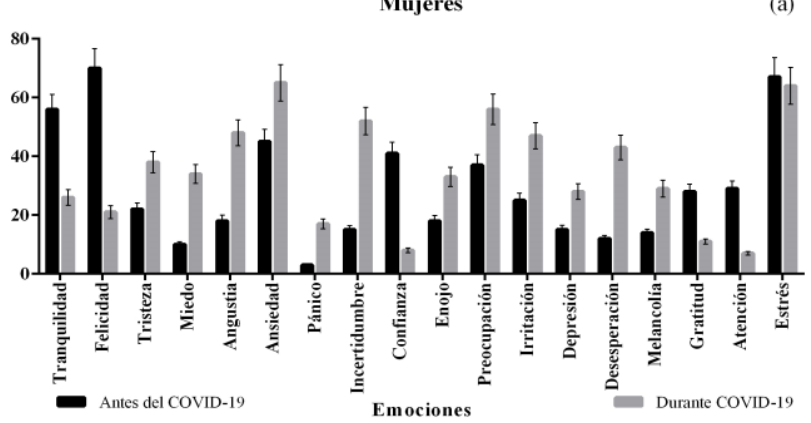

Hombres

(b)

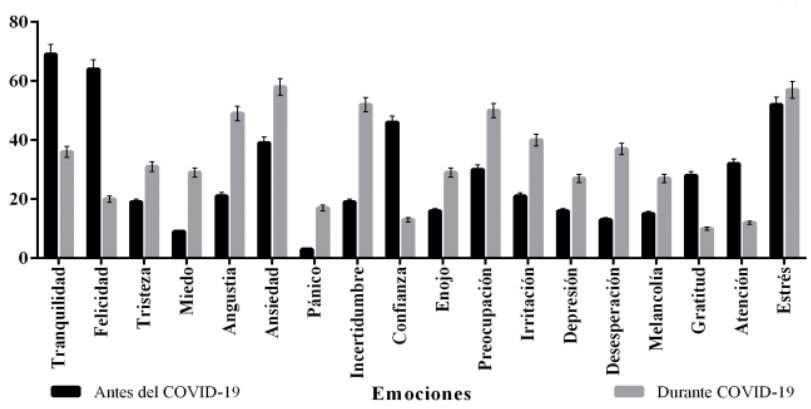

Diversa

(c)

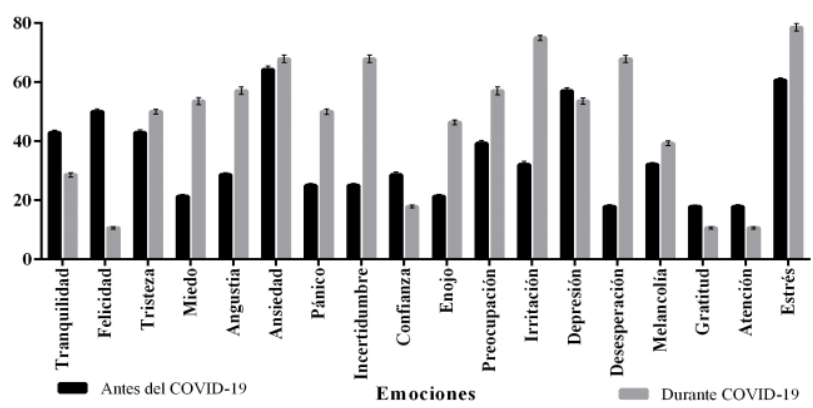




\subsection{ESCENARIOS QUE MÁS PREOCUPACIÓN E INCERTIDUMBRE GENERAN LAS MEDIDAS} POR COVID-19

Otro de los aspectos evaluados estuvo relacionado con las situaciones de mayor angustia en la comunidad estudiantil universitaria. Para más del 70\% de la población total sin importar el rango de edad, la situación financiera personal y familiar fue una de las de mayor importancia, mientras que las cuestiones de índole académica (situación académica, concluir con servicios sociales, pérdida de prácticas profesionales en clínicas y hospitales), solo resultan de interés para los participantes de menor edad (18 a 26 años) tanto en mujeres, hombres y de identidad diversa (ver Tabla 2).

Tabla 2. Escenarios que más preocupación e incertidumbre generan las medidas de contingencia por COVID-19

\begin{tabular}{|c|c|c|c|c|c|c|c|c|c|c|c|c|c|}
\hline \multirow[t]{2}{*}{$\begin{array}{c}\text { Sexo/ } \\
\text { Identidad }\end{array}$} & \multirow{2}{*}{$\begin{array}{l}\text { Edad } \\
\text { rango } \\
\end{array}$} & \multicolumn{2}{|c|}{$\begin{array}{c}\text { Situación } \\
\text { financiera } \\
\text { personal y } \\
\text { familiar }\end{array}$} & \multicolumn{2}{|c|}{$\begin{array}{l}\text { Situación } \\
\text { académica }\end{array}$} & \multicolumn{2}{|c|}{$\begin{array}{c}\text { Modificaciones } \\
\text { de planes } \\
\text { profesionales }\end{array}$} & \multicolumn{2}{|c|}{$\begin{array}{l}\text { Concluir } \\
\text { servicios } \\
\text { sociales }\end{array}$} & \multicolumn{2}{|c|}{$\begin{array}{c}\text { Perdida de } \\
\text { prácticas } \\
\text { profesionales }\end{array}$} & \multicolumn{2}{|c|}{$\begin{array}{c}\text { Modificaciones } \\
\text { de planes } \\
\text { personales }\end{array}$} \\
\hline & & $n$ & $\%$ & $n$ & $\%$ & $n$ & $\%$ & $n$ & $\%$ & $n$ & $\%$ & $n$ & $\%$ \\
\hline \multirow[t]{6}{*}{ Mujeres } & $18-20$ & 764 & 72,8 & 801 & 76,3 & 242 & 23,0 & 9 & 0,8 & 282 & 26,8 & 472 & 45,0 \\
\hline & $21-23$ & 622 & 79,8 & 602 & 77,2 & 285 & 36,5 & 122 & 15,6 & 297 & 38,1 & 384 & 49,2 \\
\hline & $24-26$ & 150 & 81,9 & 110 & 60,1 & 92 & 50,2 & 38 & 20,7 & 42 & 22,9 & 102 & 55,7 \\
\hline & 27- 29 & 50 & 81,9 & 32 & 52,4 & 26 & 42,6 & 8 & 13,1 & 8 & 13,1 & 26 & 42,6 \\
\hline & $30-32$ & 30 & 83,3 & 16 & 44,4 & 15 & 41,6 & 3 & 8,3 & 5 & 13,8 & 19 & 52,7 \\
\hline & 33 y más & 66 & 68,0 & 23 & 23,7 & 28 & 28,8 & 2 & 2,0 & 3 & 3,0 & 51 & 52,5 \\
\hline \multirow[t]{6}{*}{ Hombres } & $18-20$ & 277 & 66,9 & 342 & 82,6 & 99 & 23,9 & 12 & 2,9 & 101 & 24,4 & 191 & 46,1 \\
\hline & $21-23$ & 272 & 73,1 & 294 & 79,0 & 140 & 37,6 & 61 & 16,4 & 114 & 30,6 & 177 & 47,5 \\
\hline & $24-26$ & 61 & 70,1 & 53 & 60,9 & 38 & 43,6 & 11 & 12,6 & 17 & 19,5 & 51 & 58,6 \\
\hline & 27- 29 & 30 & 81,0 & 24 & 64,8 & 14 & 37,8 & 3 & 8,1 & 2 & 5,4 & 21 & 56,7 \\
\hline & $30-32$ & 13 & 86,6 & 8 & 53,3 & 9 & 60,0 & 2 & 13,3 & 4 & 26,6 & 9 & 60,0 \\
\hline & 33 y más & 26 & 72,2 & 11 & 30,5 & 8 & 22,2 & 1 & 2,7 & 4 & 11,1 & 15 & 41,6 \\
\hline \multirow[t]{4}{*}{ Diversa } & $18-20$ & 18 & 81,8 & 16 & 72,7 & 2 & 9,0 & - & - & 3 & 13,6 & 10 & 45,4 \\
\hline & $21-23$ & 2 & 50,0 & 3 & 75,0 & 2 & 50,0 & 1 & 25,0 & - & - & 2 & 50,0 \\
\hline & $24-26$ & 1 & 100 & 1 & 100 & 1 & 100 & 1 & 100 & 1 & 100 & 1 & 100 \\
\hline & $27-29$ & 1 & 100 & 1 & 100 & 1 & 100 & - & - & - & - & 1 & 100 \\
\hline
\end{tabular}

$\mathrm{n}=$ número de participantes; $\%=$ porcentaje

Por otro lado, las modificaciones de los planes personales resultan en los hombres de cualquier edad uno de los escenarios de mayor preocupación (48\%), el resto de las variables pueden observarse en la Tabla 2.

\subsection{ACTIVIDADES PARA MEJORAR EL ÁNIMO}

Como parte de la evaluación socioemocional, se determinaron las acciones que emplean los estudiantes de nivel superior para afrontar el distanciamiento social. Una de las estrategias que tanto mujeres como hombres emplean es el hablar con familiares y amigos (más del 50\%). El uso de redes sociales como esparcimiento es empleado por más del 50\% de los participantes y escuchar música, ver televisión o leer es la actividad de mayor preferencia (80\%). Por otro lado, se determinó que el $46 \%$ de 
Tabla 3. Actividades para mejorar el estado de ánimo

\begin{tabular}{|c|c|c|c|c|c|c|c|c|c|c|c|}
\hline \multirow[t]{2}{*}{$\begin{array}{c}\text { Sexo/ } \\
\text { Identidad }\end{array}$} & \multirow{2}{*}{$\begin{array}{l}\text { Edad } \\
\text { rango }\end{array}$} & \multicolumn{2}{|c|}{$\begin{array}{c}\text { Hablo con } \\
\text { familiares y } \\
\text { amigos }\end{array}$} & \multicolumn{2}{|c|}{$\begin{array}{c}\text { Me distraigo } \\
\text { viendo redes } \\
\text { sociales }\end{array}$} & \multicolumn{2}{|c|}{$\begin{array}{c}\text { Escucho } \\
\text { música, veo } \\
\text { TV o leo }\end{array}$} & \multicolumn{2}{|c|}{$\begin{array}{c}\text { Consumo } \\
\text { de bebida } \\
\text { alcohólica o } \\
\text { fumar }\end{array}$} & \multicolumn{2}{|c|}{$\begin{array}{c}\text { Hago } \\
\text { ejercicio }\end{array}$} \\
\hline & & $n$ & $\%$ & $n$ & $\%$ & $n$ & $\%$ & $n$ & $\%$ & $n$ & $\%$ \\
\hline \multirow[t]{6}{*}{ Mujeres } & $18-20$ & 530 & 50,5 & 681 & 64,9 & 867 & 82,6 & 54 & 5,1 & 526 & 50,1 \\
\hline & $21-23$ & 436 & 55,9 & 507 & 65,0 & 640 & 82,1 & 61 & 7,8 & 412 & 52,8 \\
\hline & $24-26$ & 103 & 56,2 & 107 & 58,4 & 150 & 81,9 & 18 & 9,8 & 84 & 45,9 \\
\hline & 27- 29 & 40 & 65,5 & 32 & 52,4 & 41 & 67,2 & 7 & 11,4 & 28 & 45,9 \\
\hline & $30-32$ & 24 & 66,6 & 23 & 63,8 & 25 & 69,4 & 3 & 8,3 & 14 & 38,8 \\
\hline & 33 y más & 60 & 61,8 & 42 & 43,3 & 70 & 72,1 & 7 & 7,2 & 47 & 48,4 \\
\hline \multirow[t]{6}{*}{ Hombres } & $18-20$ & 208 & 50,2 & 276 & 66,6 & 359 & 86,7 & 43 & 10,3 & 206 & 49,7 \\
\hline & $21-23$ & 177 & 47,5 & 214 & 57,5 & 294 & 79,0 & 67 & 18,0 & 172 & 46,2 \\
\hline & $24-26$ & 44 & 50,5 & 45 & 51,7 & 65 & 74,7 & 24 & 27,5 & 42 & 48,2 \\
\hline & 27- 29 & 17 & 45,9 & 21 & 56,7 & 25 & 67,5 & 2 & 5,4 & 13 & 35,1 \\
\hline & $30-32$ & 8 & 53,3 & 9 & 60,0 & 14 & 93,3 & 7 & 46,6 & 7 & 46,6 \\
\hline & 33 y más & 29 & 80,5 & 16 & 44,4 & 24 & 66,6 & 4 & 11,1 & 12 & 33,3 \\
\hline \multirow[t]{4}{*}{ Diversa } & $18-20$ & 6 & 27,2 & 12 & 54,5 & 19 & 86,3 & 7 & 31,8 & 5 & 22,7 \\
\hline & $21-23$ & 2 & 50,0 & 2 & 50,0 & 3 & 75,0 & - & - & 2 & 50,0 \\
\hline & $24-26$ & - & - & - & - & 1 & 100 & 1 & 100 & 1 & 100 \\
\hline & 27- 29 & - & - & 1 & 100 & 1 & 100 & 1 & 100 & - & - \\
\hline
\end{tabular}

\section{DISCUSIÓN}

El propósito de esta investigación fue identificar y analizar los procesos socioemocionales de estudiantes universitarios por las medidas de control sanitarias en la Jornada de Sana Distancia implementada por la Secretaría de Salud en México por COVID-19. La exploración psicológica de las alteraciones emocionales en situaciones de cuarentena, confinamiento, distanciamiento y aislamiento social son escasas, más aún entre la población de adultos jóvenes universitarios. Investigaciones en el campo de la salud mental señalan la necesidad de incluir el peso de las determinantes sociales en los efectos psicoemocionales por la pandemia por COVID-19 dentro de los cuales se incluyen los procesos socioemocionales que muestran la relación entre los ejes de estructuración social y la vida sensible de los sujetos (Medina-Mora, 2015).

Los resultados preliminares de este trabajo sobre la manifestación de las emociones antes y durante el confinamiento sugieren la existencia de procesos socioemocionales compartidos entre los sexos en la comunidad estudiantil universitaria. Es decir, mujeres, hombres e identidad diversa experimentan el 
mismo tipo de emociones asociadas con el malestar y el bienestar en sus escenarios sociales. La información obtenida en esta investigación mostró menos asimetrías sociales intergenéricas basadas en la vida emocional. Los resultados evidencian que la experiencia emocional también puede convertirse en un indicador social de las desigualdades entre los géneros como se ha demostrado en otras investigaciones (López, 2011; Hochschild, 1979).

Los hallazgos de esta investigación mostraron que la comunidad estudiantil más interesada en participar fue la de nivel licenciatura. Donde las mujeres tuvieron mayor interés que los hombres en responder un cuestionario enfocado en explorar la vida emocional como el MPE-COVID-19. Tal comportamiento se puede explicar en función de los roles sociales relacionados con las identidades sexo genéricas, en las que la expresión de las emociones por las mujeres es parte de la permisividad social (Lutz, 1986). En consonancia con la premisa que vincula la expresión emocional con la identidad sexo genérica se identificó que las mujeres emplean un mayor léxico de emociones asociadas con el malestar físico y psicológico, mientras que los hombres emplean la mitad de ese vocabulario. Ese dato cualitativodescriptivo puede interpretarse como parte de los efectos culturales que influyen en el aprendizaje de los procesos socioemocionales que coloca a las mujeres como más emocionales y a los hombres como más racionales (López, 2011; Hochschild, 1979). Por otro lado, la participación de la población con identidad diversa fue menor que la de hombres y mujeres, aun cuando la FES-Iztacala se caracteriza por la manifestación activa de esta comunidad (Gaceta Iztacala UNAM, 2018).

Posterior al inicio de la Jornada de Sana Distancia hubo una disminución en la expresión de emociones de bienestar e incremento en las relacionadas con el malestar en toda la población participante; el incremento de las emociones de malestar fue coincidente con hallazgos de otros estudios, donde de igual manera identificaron enojo, miedo y ansiedad como resultado del aislamiento social (MendozaVelásquez, 2020; Brooks et al., 2020). La coexistencia de ambos tipos de emociones podría parecer contradictoria. Esto se explica a través de la observación de los estilos de vida de la comunidad estudiantil, los cuales incluyen demandas académicas, familiares y en algunos casos laborales generando ansiedad, preocupación, irritación y estrés. Sin embargo, lo que muestran los resultados de este estudio es que la sociabilidad positiva de las y los jóvenes en esos mismos escenarios refuerza sus vínculos eróticoafectivos, de amistad, familiares, laborales y profesionales, entre otros. Los cuales se constituyen en fuentes de confianza, tranquilidad, felicidad, gratitud y satisfacción contrarrestando las emociones de malestar que viven en el día a día. La sociabilidad entre pares en la comunidad universitaria funge como un regulador emocional en beneficio de un equilibrio en la salud mental (Martell, 2020).

En consonancia con Brooks et al. (2020), el escenario financiero fue el que mayor incertidumbre y angustia generó en la comunidad estudiantil independientemente de edad y sexo. Las afectaciones 
financieras familiares y personales de estudiantes están conllevando a que realicen actividades remuneradas para apoyar los ingresos económicos familiares. Originando como consecuencia, la interferencia en sus laborales académicas; enfrentando la paradójica disyuntiva de mantener sus estudios en línea o trabajar para pagar los servicios de internet, lo que les permitiría, sobre todo a los de últimos semestres, concluir satisfactoriamente sus cursos y licenciaturas.

Relacionado con lo anterior, los cambios en los planes personales en los hombres de todas las edades resultan ser una de las situaciones de más preocupación. Al respecto, se ha reportado que las modificaciones en las dinámicas familiares, económicas, laborales y sociales fungen como detonantes de ansiedad y depresión (Piña-Ferrer, 2020).

La comunidad de estudiantes mostró un interés mayor por actividades individuales y de socialización virtual, a través de redes sociales (Facebook, Instagram, WhatsApp) para gestionar sus estados emocionales indistintamente del sexo y edad. Desde el comienzo de la pandemia COVID-19, el Boletín BIREME (2020) informó del aumento del tiempo en el uso de redes sociales en la población de todas las edades y segmentos de la sociedad, lo cual coincide con los hallazgos de esta investigación.

El aumento en el consumo de alcohol, tabaco y marihuana entre los hombres y la población diversa en el estudio muestra que su uso incrementa en momentos de crisis y se llega a considerar como una práctica de reducción de la ansiedad (Cano-Vindel et al., 1994). Además, el consumo de sustancias también se asocia con una cultura de género, en la que se promueve una masculinidad hegemónica con dificultades para expresar sus emociones, la práctica de conductas de riesgo y consumo de alcohol (Ramírez, 2005; Rivas-Sánchez, 2005; Enríquez \& López, 2018).

Los resultados preliminares de esta investigación permitieron identificar las limitaciones del estudio en términos de la representatividad de la comunidad con "identidad diversa", el análisis fenomenológico de la vida emocional que incluya relaciones y escenarios sociales diversos no contemplados por el MPE-COVID-19. Este aspecto puede ser atendido a través de metodologías cualitativas como entrevistas en profundidad o grupos focales para contar con mayores evidencias para establecer relaciones directas entre la identidad sexo-genérica, la vida emocional y los factores sociales que expliquen las alteraciones emocionales en momentos de emergencia como la vivida por la pandemia COVID-19. Los procesos socioemocionales de estudiantes universitarios, durante la Jornada de Sana Distancia, se gestionaron en función de las variables de estratificación social, las cuales definen sus vidas y genera impacto en el funcionamiento psicofisiológico de las emociones.

Agradecimientos: El artículo es un producto directo del Proyecto: Características psicosociales y procesos socioemocionales en comunidades estudiantiles universitarias frente al aislamiento sanitario por COVID 19: hacia una política institucional del cuidado, autocuidado y autoatención financiado por el 
Programa de Apoyo a Proyectos de Investigación e Innovación Tecnológica (PAPIIT) de la Dirección General del Personal Académico de la Universidad Nacional Autónoma de México (DGAPA-UNAM), registro IN301021. 


\section{REFERENCIAS}

Asociación Médica Mundial (2013). Declaración de Helsinki de la AMM Principios éticos para las investigaciones médicas en seres humano. Helsinki: Asociación Médica Mundial. https://www.wma.net/es/policies-post/declaracion-de-helsinki-de-la-amm-principios-eticos-para-lasinvestigaciones-medicas-en-seres-humanos/

Bericat, A. E. (2018). Excluidos de la felicidad. La estratificación social del bienestar emocional en España. Centro de Investigaciones Sociológicas.

Boletín BIREME. (2020). Las redes sociales y COVID-19: la contribución de BIREME. Boletín BIREME, 43. http://boletin.bireme.org/2020/05/02/las-redes-sociales-y-covid-19-la-contribucion-de-bireme/

Brooks, S. K., Webster, R. K., Smith, L. E., Woodland, L., Wessely, S., Greenberg, N., \& Rubin, G.J. (2020). The psychological impact of quarantine and how to reduce it: rapid review of the evidence. Lancet, 395(10227), 912-20.

Cano-Vindel, A., Miguel-Tobal, J. J., González, H., \& Iruarrizaga, I. (1994). El afrontamiento de la ansiedad en las drogodependencias. Anales de Psicología, 10(2), 145-156.

Enríquez, R., \& López, O. (2018). Masculinidades, familias y comunidades afectivas. Instituto Tecnológico de Estudios Superiores de Occidente, Universidad Jesuita de Guadalajara, Facultad de Estudios Superiores Iztacala, Universidad Nacional Autónoma de México.

Gaceta Iztacala UNAM. (2018). La FES Iztacala instala baños mixtos. Gaceta Iztacala UNAM. https://fenix.iztacala.unam.mx/?p=16818

Hochschild, A. (1979). Emotion Work, Feeling Rules, and Social Structure. American Journal of Sociology, 85(3), 551-575.

Ledermann, W. (2003). El hombre y sus epidemias a través de la historia [Edición aniversario]. Revista chilena de infectología, 20, 13-7. http://dx.doi.org/10.4067/S0716-10182003020200003

Ley Federal de Protección de Datos Personales en Posesión de los Particulares, 2010. Diario Oficial de la Federación (México). http://www.diputados.gob.mx/LeyesBiblio/pdf/LFPDPPP.pdf

Ley General de Salud, Art. 79. Reformas del 24 de enero de 2020. Diario Oficial de la Federación (México). https://www.dof.gob.mx/nota_detalle.php?codigo=5584753\&fecha=24/01/2020

López, O. (2011). La pérdida del paraíso. El lugar de las emociones en la sociedad mexicana entre los siglos XIX y XX. Facultad de Estudios Superiores Iztacala, Universidad Nacional Autónoma de México. López, O. (2019). Extravíos del alma mexicana. Patologización de las emociones en los diagnósticos psiquiátricos (1900-1940). Facultad de Estudios Superiores Iztacala, Universidad Nacional Autónoma de México.

López, O. (2020, junio 26). La importancia de los aspectos psicosociales y los procesos socioemocionales por las medidas sanitarias de distanciamiento social COVID-19. Gaceta Iztacala UNAM. https://fenix.iztacala.unam.mx/?p=28583 
Lutz, C. (1986). Emotion, thought, and estrangement: emotion as a cultural category. Cultural Anthropology, 1(3), 287-309.

Martell, E. O. (2020). Afectividades emergentes en los encuentros situados en la Facultad de Ciencias Políticas y Sociales de la UNAM. En O. López \& R. Enríquez (Coord.) Juventudes y emociones desde la perspectiva sociocultural (pp. 71-96). Facultad de Estudios Superiores Iztacala, Universidad Nacional Autónoma de México, Instituto Tecnológico de Estudios Superiores de Occidente, Universidad Jesuita de Guadalajara.

Medina-Mora, M. E., Sarti-Gutiérrez, E. J., Real-Quintanar, T., (Eds.). (2015). La depresión y otros trastornos psiquiátricos. Academia Nacional de Medicina-CONACYT.

Mendoza-Velásquez, J. J. (2020, 10 de marzo). Impacto de la COVID-19 en la salud mental. Medscape. https://espanol.medscape.com/verarticulo/5905131

Norma Oficial Mexicana NOM-012-SSA3-2012, 2013. Que establece los criterios para la ejecución de proyectos de investigación para la salud en seres humanos. Diario Oficial de la Federación (México). http://dof.gob.mx/nota_detalle.php?codigo=5284148\&fecha=04/01/2013

Piña-Ferrer L. (2020). El COVID 19: Impacto psicológico en los seres humanos. Revista Arbitrada Interdisciplinaria de Ciencias de la Salud. Salud y Vida, 4(7), 188-199. https://dialnet.unirioja.es/servlet/articulo?codigo=7407744

Ramírez, J. C. (2005). Madejas entreveradas. Masculinidad, violencia y poder. Guadalajara. Plaza y Valdés y Universidad de Guadalajara.

Riesgo, M. (2020, abril 14). El confinamiento prolongado puede aumentar el riesgo de suicidio. Gaceta Médica. https://gacetamedica.com/investigacion/el-confinamiento-prolongado-puede-aumentar-elriesgo-de-suicidio/

Rivas-Sánchez, H. E. (2005). ¿El varón como factor de riesgo? Masculinidad y mortalidad por accidentes y otras causas violentas en la sierra de Sonora. Estudios Sociales, 13(26), 28-65.

Rodríguez-Yunta, E. (2016). Determinantes sociales de la salud mental. Rol de la religiosidad. Persona y Bioética, 20(2), 192-204. https://dialnet.unirioja.es/servlet/articulo?codigo=5744068

Rubin GJ, Wessely S. The psychological effects of quarantining a city. BMJ, 368, artículo m313. doi:10.1136/bmj.m313

Sánchez-Villena, A. R., \& de la Fuente-Figuerola, V. (2020). COVID-19: cuarentena, aislamiento, distanciamiento social y confinamiento ¿son lo mismo? An Pediatr, 93(1), $73-4$. https://doi.org/10.1016/j.anpedi.2020.05.001

Sol-Pastorino, M., Vanegas-López, J., \& Florenzano-Urzúa, R. (2017). Mental health with a gender perspective. Salud Pública de México, 59(6), 601-602. https://doi.org/10.21149/8508

Thoits, P. (1990). Emotional Deviance: Research Agendas. En T. D. Kemper (Ed.). Research Agendas in the Sociology of Emotions (pp. 180-203). State University of New York Press. 
Urbina, M. (2016). Los determinantes sociales de la salud y de la equidad en salud. Academia Nacional de Medicina, CONACyT. 